\title{
L'empreinte génomique : complémentarité fonctionnelle des deux génomes parentaux
}

Les génomes d'origine paternelle et maternelle ne sont pas équivalents et jouent un rôle complémentaire au cours du développement des mammifères. Le fonctionnement différentiel des deux génomes parentaux est la conséquence d'une modification épigénétique - l'empreinte parentale - apposée sur le génome au cours de la gamétogenèse. Des expériences de création d'embryons constitués de deux génomes mâles (androgénotes) ou femelles (gynogénotes) montrent que les premiers participent de manière prédominante au développement des annexes extra-embryonnaires et les seconds à celui de l'embryon. Au sein de l'embryon, cependant, certains tissus, tel le muscle, nécessitent pour leur développement la présence du génome mâle. Les conséquences de l'empreinte génomique parentale peuvent aussi être appréciées au niveau de l'expression de transgènes, variable selon qu'ils sont transmis par les gamètes mâles ou femelles, et du phénotype de disomies uniparentales ou de mutations, là encore parfois différentes en fonction du parent dont elles proviennent.

\section{Andras Paldi Jacques Jami}

\footnotetext{
ADRESSES

J. Jami : direcleur de recherche à l'Inserm. Institut Jacques-Monod, Inserm U. 257, 2 , place Jussieu, 75251 Paris, France. A. Paldi : chargé de recherche. Adresse permanente : Agricultural Biotechnology Center, 2101 Gödöllö, pf. 170, Hongrie.
}

$m / s n^{\circ} 3$, vol. 7, mars 91

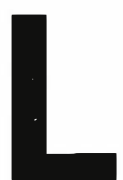

e génome des animaux est diploïde, et les gènes portés par les chromosomes autosomes sont représentés par deux copies homologues, l'une d'origine paternelle, l'autre d'origine maternelle. Selon la vision classique de l'hérédité génétique, les caractères d'un individu sont déterminés par l'ensemble de l'information génétique transmise par les deux parents et les deux copies du même gène apportent des informations équivalentes, indépendamment de leur origine paternelle ou maternelle : ils sont interchangeables. Mais des études embryologiques et génétiques récentes [1-3], qui ont déjà été pré- sentées aux lecteurs de médecine/sciences [4], ont démontré que ce n'est pas toujours le cas. Une partie du génome fonctionne différemment selon qu'il est transmis par le père ou par la mère. Au niveau de l'organisme, cela signifie que les gènes porteurs de cette empreinte parentale doivent être représentés dans le zygote des mammifères à la fois par une copie (allèle) maternelle et par une copie paternelle, pour assurer le développement normal de l'individu. La différence fonctionnelle des deux allèles parentaux est la conséquence des modifications apposées sur les gènes impliqués au cours de la différenciation des cellules germinales 


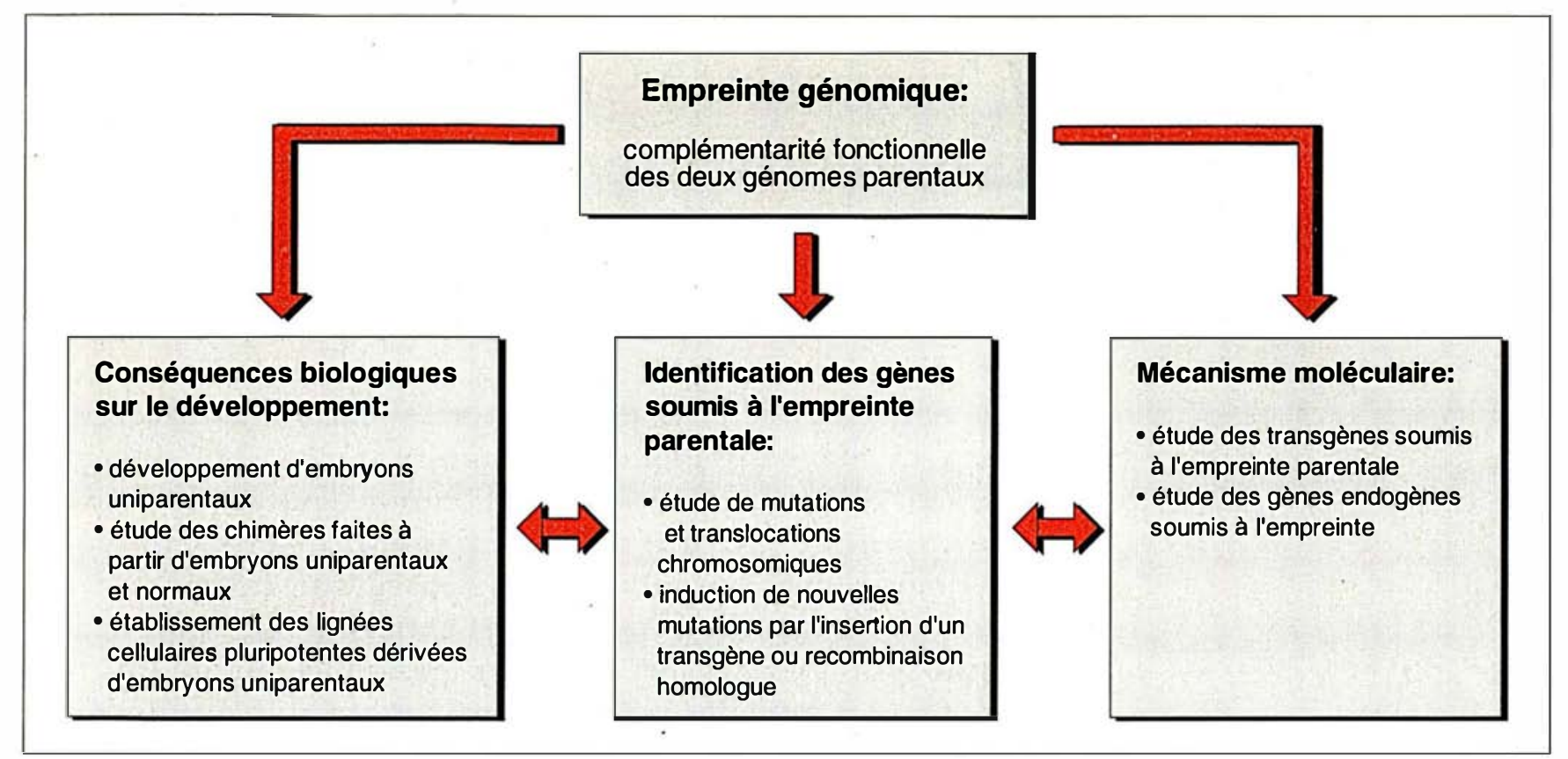

Figure 1. Différentes approches expérimentales de l'étude de l'empreinte génomique.

mâles et femelles. Cette empreinte est réversible. Le gène considéré passe sans empreinte à la génération suivante quand il est transmis par un gamète du sexe opposé (du père à sa fille ou de la mère à son fils).

Ce phénomène est distinct de l'expression liée au sexe de gènes portés par le chromosome $\mathrm{X}$, qui est due à l'haploïdie physique chez le mâle, ou fonctionnelle chez la femelle, de ce chromosome sexuel. Il est également distinct de l'inactivation préférentielle du chromosome $\mathrm{X}$ d'origine paternelle observée dans les tissus extra-embryonnaires [5].

On ignore le nombre de gènes qui sont soumis à cette empreinte parentale. Il semble qu'il y en ait sur presque tous les chromosomes de la souris, mais, jusqu'ici, seul l'un d'entre eux a été identifié [6].

\section{A la recherche des gènes marqués}

Malgré les nombreuses études effectuées au cours des dernières années, les renseignements obtenus sont encore très fragmentaires et les questions principales restent encore sans réponse. Ces questions portent sur l'identité des gènes soumis à l'empreinte parentale, sur les fonc- également sur le mécanisme moléculaire de ce phénomène.

Plusieurs stratégies sont employées par les chercheurs pour aborder le problème (figure 1). La première est une approche déductive, elle consiste à étudier les capacités de développement des embryons uniparentaux ou des cellules qui en sont dérivées. Ces observations permettent d'identifier les étapes du développement qui sont contrôlées par les gènes porteurs d'une empreinte paternelle ou maternelle. Une autre stratégie essaie de tirer avantage de l'existence de rares mutations spontanées ou induites artificiellement pour identifier les

\section{* GLOSSAIRE *}

Androgénote : embryon se développant à partir d'un ouf dont le pronucléus femelle a été énucléé et remplacé par un second pronucléus mâle.

Gynogénote : embryon se développant à partir d'un ouf fécondé dont le pronucléus mâle a été énucléé et remplacé par un second pronucléus femelle.

Parthénote : embryon développé à partir d'un œuf non fécondé. gènes en question. Mais seul le clonage des gènes soumis à l'empreinte permettra de caractériser le mécanisme responsable de ce phénomène.

\section{Implications biologiques de l'empreinte parentale}

Les études portant sur le développement des embryons uniparentaux ont orienté vers le caractère complémentaire indispensable des deux génomes parentaux (figure 2). Les embryons porteurs d'un génome diploïde exclusivement d'origine maternelle (gynogénotes et parthénotes) ou d'un génome diploïde exclusivement d'origine paternelle (androgénotes) sont incapables de se développer normalement jusqu'à terme [1, 3]. Leur développement s'arrête peu après l'implantation dans l'utérus. Toutefois, les potentialités de développement de ces deux types d'embryons sont différentes [7]. Les gynogénotes sont capables de se développer jusqu'au stade des somites et ils forment un embryon morphologiquement normal. Mais les tissus extraembryonnaires - trophoblaste et sac vitellin - restent sous-développés. $\mathrm{Au}$ contraire, les androgénotes forment des tissus extra-embryonnaires normaux mais l'embryon reste rudimentaire. Les phénotypes observés 
dans les deux cas suggèrent que les rôles joués par les génomes d'origine maternelle et paternelle sont complémentaires : le premier est nécessaire au développement de l'embryon et le deuxième est indispensable à la formation des tissus extra-embryonnaires. Une autre observation résulte du développement de souris chimères réalisées par agrégation de deux embryons. Lorsqu'on agrège deux embryons normaux, on peut détecter des cellules des deux embryons dans tous les tissus constituant l'embryon chimère, les annexes extraembryonnaires et l'animal développé (figure 3, p. 251). En revanche, les embryons chimériques formés par l'agrégation d'un embryon gynogénote et d'un embryon androgénote ne peuvent pas se développer jusqu'au terme de la gestation, confirmant que les deux génomes parentaux doivent être présents ensemble dans chacune, ou dans la majorité des cellules de l'embryon [8]. La distribution des deux types de cellules dans les fotus chimériques est très caractéristique. Les cellules provenant de l'embryon parthénote (ou gynogénote) colonisent de préférence l'embryon, et les cellules d'origine androgénétique ne forment que les annexes extra-embryonnaires.

Cependant, quand ils sont fusionnés avec des embryons normaux [9-11], les androgénotes et les gynogénotes peuvent contribuer à la différenciation des tissus des souris chimères. Comme on pouvait s'y attendre, les cellules gynogénétiques sont très vite éliminées dans les tissus extraembryonnaires de la chimère, mais elles persistent dans l'embryon. Toutefois, leur participation tend à diminuer vers la fin de la gestation [12], et elles n'ont pas la même potentialité pour participer à la formation des différents tissus. Dans certains tissus comme les dérivés neuro-ectodermiques, la contribution gynogénétique peut atteindre 50-60 \% à la naissance. En revanche, d'autres tissus, comme le muscle squelettique ou le foie, ne contiennent presque jamais de cellules provenant du composant gynogénote $[13,14]$. Les chimères ayant une forte participation gynogénétique sont plus petites que les chimères ayant une contribution faible et que les souris normales [15].

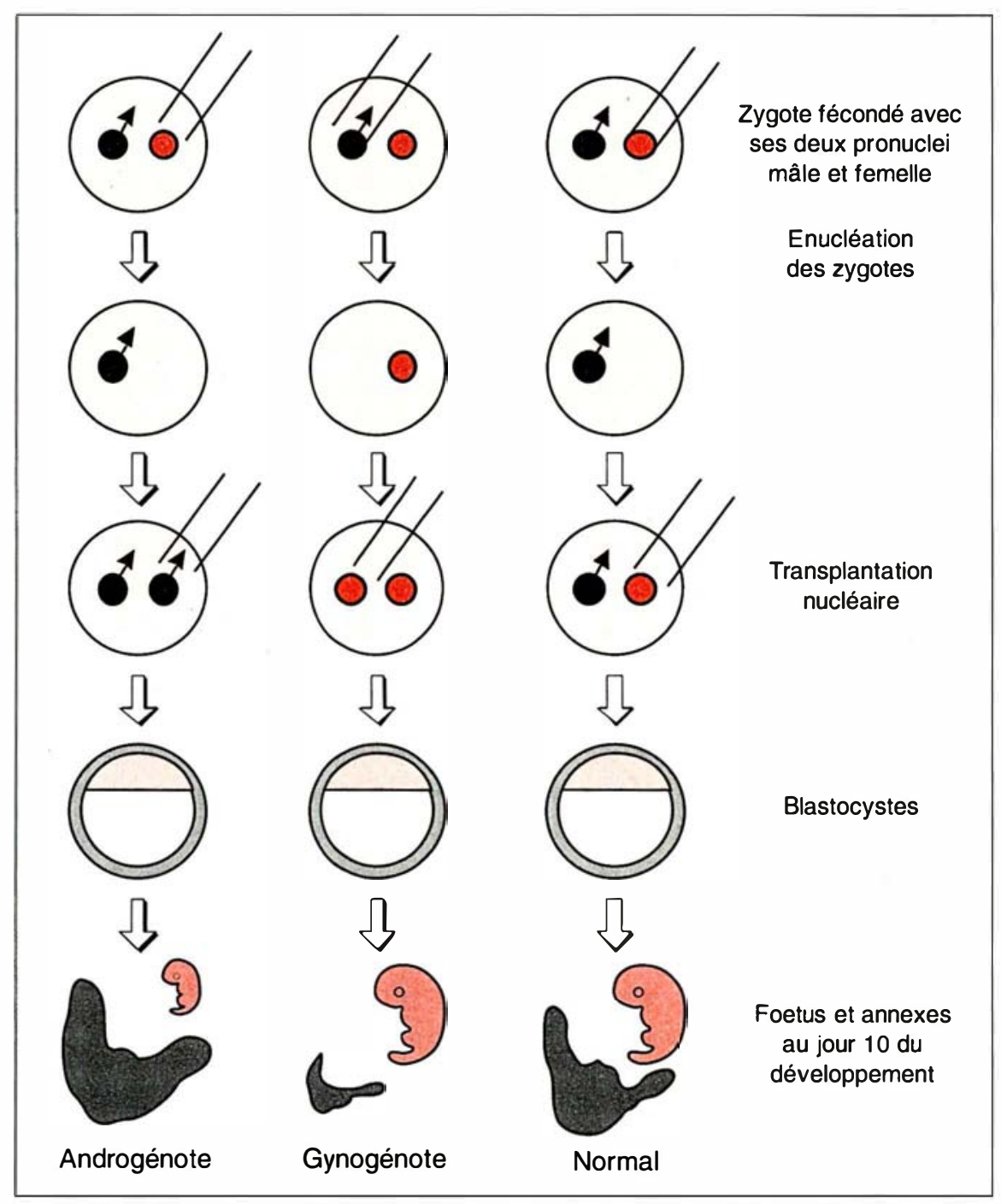

Figure 2. Complémentarité des deux génomes parentaux dans le développement ontogénique. Après fécondation d'un œuf de souris, on peut enlever à l'aide d'une micropipette un pronucléus et le remplacer par le pronucléus mâle ou femelle d'un autre zygote. On obtient ainsi les premières étapes du développement d'un embryon diploïde dont le génome est exclusivement d'origine mâle (androgénote), ou exclusivement d'origine femelle (gynogénote), ou d'origine biparentale (normal). Les androgénotes et les gynogénotes sont incapables de se développer jusqu'au terme de la gestation après transplantation dans l'oviducte d'une femelle pseudogestante. L'androgénote forme des tissus extra-embryonnaires normaux ou hypertrophiés, mais l'embryon reste rudimentaire. Le gynogénote forme un embryon morphologiquement normal jusqu'au stade des somites, mais les tissus extra-embryonnaires restent sousdéveloppés. 
Il y a donc, au cours du développement, une pression de sélection contre les cellules d'origine parthénogénétique. Cette sélection a lieu probablement dans tous les tissus des chimères, mais elle est plus forte dans certains tissus, comme le muscle squelettique ou le foie, et moins forte dans d'autres tissus, comme le cerveau [16]. Les fœtus chimériques androgénote $\leftrightarrow$ normal ont un phénotype différent des fœus chimériques parthénote ou gynogénote $\leftrightarrow$ normal que nous venons de décrire. Les cellules androgénétiques ne participent pas à la formation de l'embryon: on les retrouve exclusivement dans les tissus extraembryonnaires [11].

\section{RÉFÉRENCES}

1. McGrath J, Solter D. Completion of mouse embryogenesis requires both the maternal and paternal genomes. Cell 1984 ; $37: 179-83$.

2. Cattanach BM, Kirk M. Differential activity of maternally and paternally derived chromosome regions in mice. Nature 1985 ; 315: 496-8.

3. Surani MAH, Barton SC, Norris ML. Development of reconstituted eggs suggest imprinting of the genome during gametogenesis. Nature $1984 ; 308: 548-50$.

4. Babinet C, Barra J, Renard JP. Le marquage et l'expression diffćrentiels des génomes paternel et maternel. médecine/sciences $1989 ; 5: 8-15$.

5. Monk M. Changes in DNA methylation during mouse embryonic development in relation to $\mathrm{X}$-chromosome activity and imprinting. Phil Trans $R$ Soc Lond B 1990 ; 326 : 299-312.

6. Barlow DP, Stöger R, Herrmann BG, Saito K, Schweifer N. The mouse insulinlike growth factor type-2 receptor is imprinted and closely linked to the Tme locus. Nature 1991 ; 349 : 84-7.

7. Barton SC, Surani MAH, Norris ML. Role of paternal and maternal genomes in mouse development. Nature 1984; 311 : 374-6.

8. Surani MAH, Barton SC, Norris ML. Influence of parental chromosomes on spatial specificity in androgenetic-parthenogenetic chimaeras in mouse. Nature 1987 ; 326 : 395-7.

9. Stevens LC. Totipotent cells of parthenogenetic origin in a chimaeric mouse. Nalure 1978 ; 276 : 266-7.

10. Stevens LC, Varnum DS, Eicher EM. Viable chimaeras produced from normal and parthenogenetic mouse embryos. Nature
Récemment, une équipe américaine a établi des lignées de cellules ES (ES = embryonic stem cells, cellules souches de l'embryon) à partir d'embryons androgénétiques [17]. Les cellules ES sont dérivées de la masse cellulaire interne du blastocyste (pour revue, voir [18]). Elles peuvent être maintenues en culture pendant plusieurs générations en état non différencié tout en conscrvant leur capacité de se différencier après avoir été injectées dans un blastocyste receveur. Par aillcurs, l'inoculation souscutanée de cellules ES à des souris histocompatibles aboutit au développement de tératocarcinomes, tumeurs qui comportent de nombreux tissus différenciés dérivés des trois feuillets embryonnaires primitifs (figure 4). Sur la base de nos connaissances du développement des androgénotes, le fait de pouvoir obtenir une telle lignée ES à partir d'un embryon androgénétique [17] était inattendu. Encore plus surprenant, ces cellules sont capables de coloniser tous les tissus de la souris chimère obtenue par leur injection dans des blastocystes. Ces chimères présentent des malformations squelettiques très sévères, plus particulièrement une hyperplasie du cartilage. Quand les cellules ES androgénétiques sont injectées sous la peau d'une souris normale, elles développent des tumeurs. Mais contrairement aux tumeurs provenant des cellules ES normales ou parthénogénétiques, elles sont composées presque uniquement de muscle [17]. L'ensemble de ces résultats suggère que le génome des cellules ES andro- génétiques retient en partie l'empreinte de type paternel et que les phénotypes des souris chimères et des tumeurs sont la conséquence directe soit de l'inactivation soit de la surexpression de gènes porteurs du marquage paternel. Par exemple, certains gènes régulateurs de la différenciation musculaire et squelettique pourraient être soumis à l'empreinte parentale, ce qui expliquerait, d'une part, les malformations squelettiques et le développement des tumeurs musculaires par les cellules androgénétiques, et, d'autre part, l'incapacité des cellules gynogénétiques de se différencier en muscle dans les embryons chimériques. L'existence des cellules ES androgénétiques et gynogénétiques rendra possible l'analyse moléculaire de cette hypothèse et, plus généralement, de l'empreinte parentale.

Une autre approche pour élucider les conséquences biologiques de l'empreinte parentale est l'étude de souris porteuses de certains types de translocations chromosomiques [2, $19,20]$. Les souris hétérozygotes pour une translocation réciproque (figure 5, p. 252) produisent plusieurs types de gamètes. A cause d'une non-disjonction des chromosomes appariés lors de la méiose, certains gamètes gardent deux copies d'un fragment chromosomique alors que d'autres ne possèdent pas ce fragment. En étudiant les descendants de deux parents hététozygotes pour un type de translocation réciproque, on devrait observer, avec une fréquence calculable, l'apparition de souris parfaitement diploïdes mais qui possèdent un fragment chromosomique provenant d'un seul des deux parents (combinaison de gamètes $\mathrm{AB}$ ou $\mathrm{CD}$ de la figure 5). Mais ce n'est pas toujours le cas! L'étude systématique des souris porteuses de différents types de translocations réciproques a révélé que certaines régions chromosomiques ne sont pas fonctionnellement équivalentes selon que ces régions ont été héritées d'un parent ou de l'autre. Dans certains cas, la "non-complémentation" est totale. Par exemple, la duplication maternelle de la région proximale du chromosome 2 accompagnée de l'absence de la région homologue d'origine paternelle est létale, mais la duplica- 


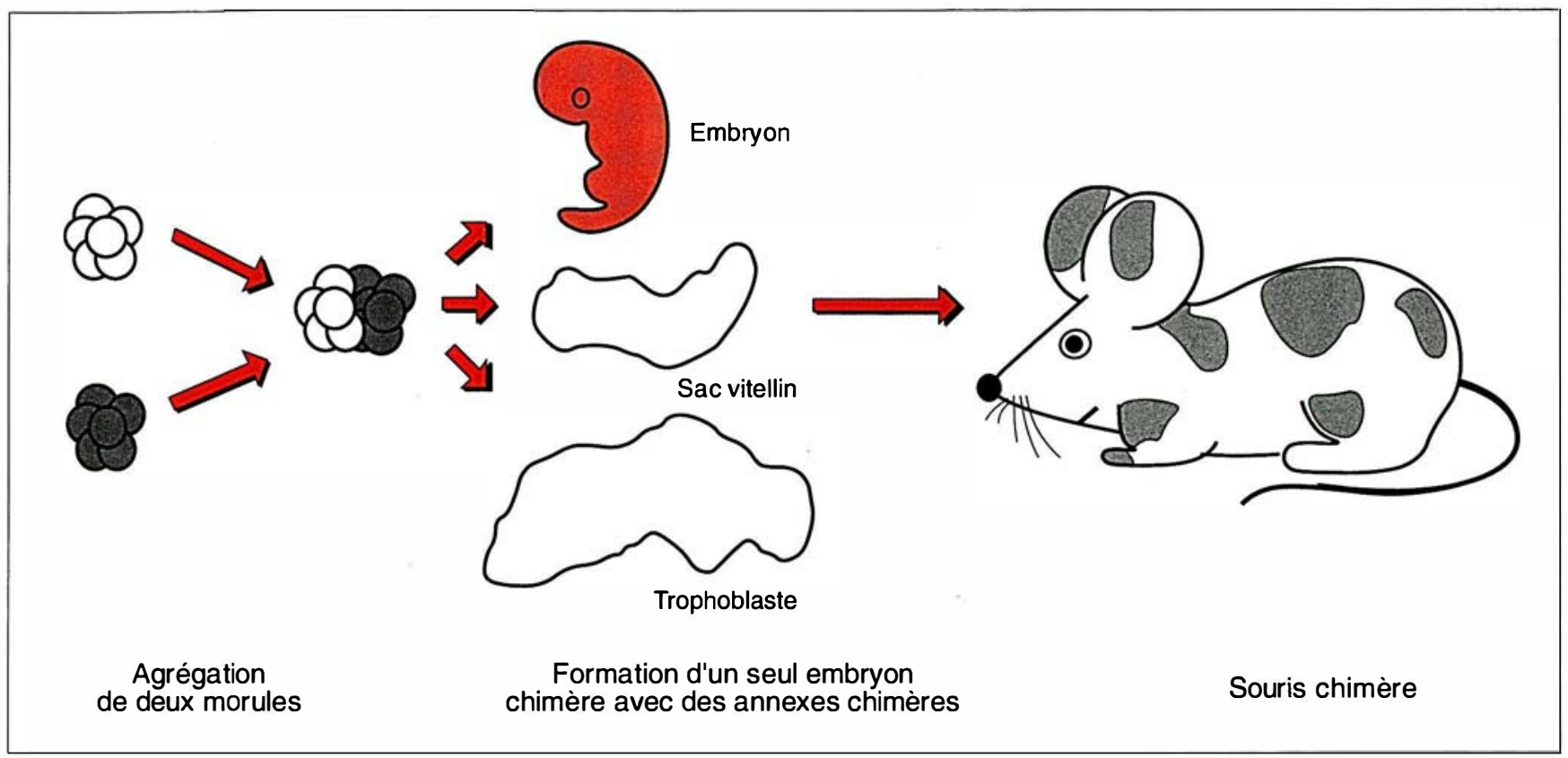

Figure 3. Obtention d'une souris chimère par agrégation de deux embryons. L'agrégation de deux embryons au stade 8 cellules aboutit par fusion au développement d'un embryon chimère unique dans lequel coexistent les cellules diploïdes des deux embryons d'origine. Le développement de cet embryon aboutit à la formation d'une souris chimère dont chaque organe peut être constitué d'une mosaïque de cellules des deux composants.

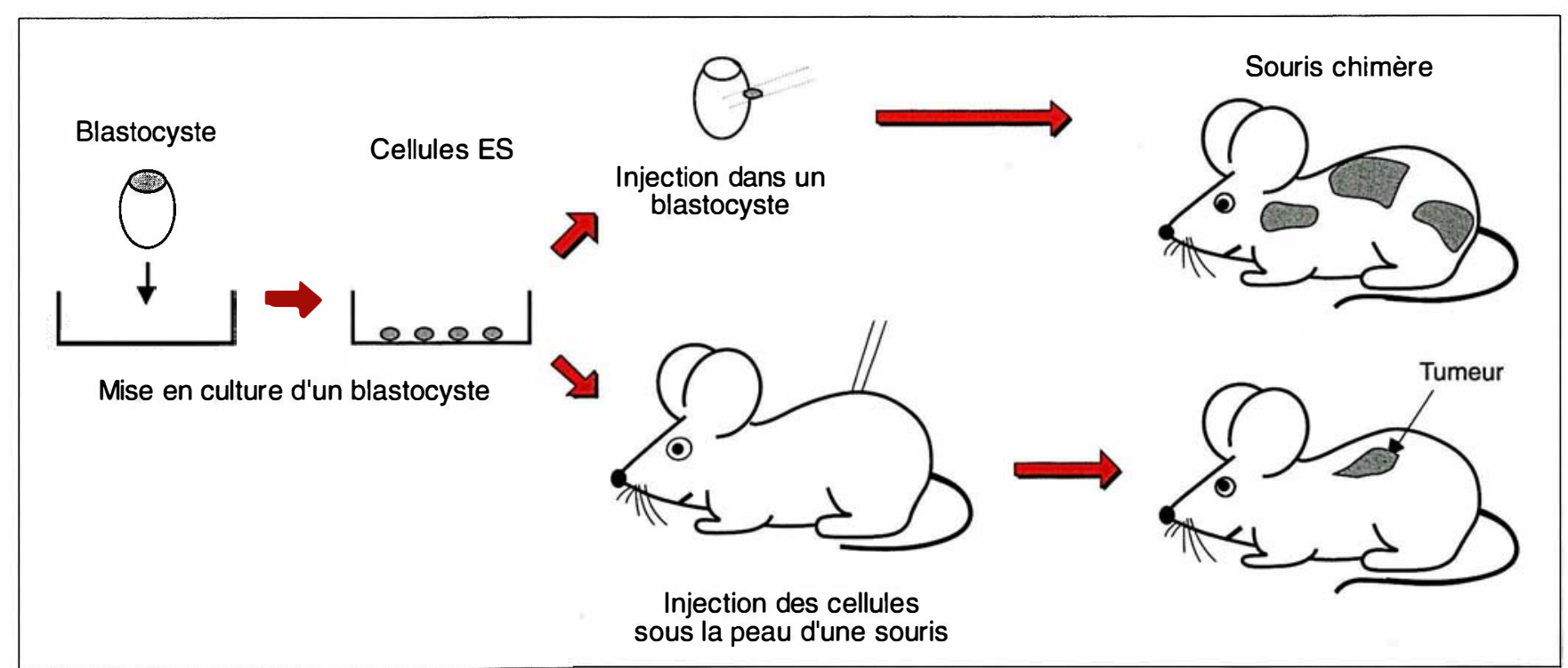

Figure 4. Les cellules ES sont des cellules embryonnaires totipotentes en culture. On peut obtenir par prolifération en culture une très grande population de cellules ES à partir des quelques dizaines de cellules de la masse interne du blastocyste. L'injection de cellules ES dans la cavité d'un blastocyste hôte aboutit au développement d'une souris chimère. Celle-ci peut former des gamètes dérivés de la cellule ES, et ainsi contribuer au développement d'animaux normaux dérivés de cellules en culture. L'inoculation sous-cutanée de cellules ES à une souris aboutit au développement de tératocarcinomes, tumeurs malignes contenant des tissus différenciés dérivés des trois feuillets embryonnaires primitifs. 


\section{RÉFÉRENCES}

11. Surani MAH, Barton SC, Howlett SK Norris ML. Influence of chromosomal determinants on development of androgenetic and parthenogenetic cells. Development 1988; 103: 171-8.

12. Nagy A, Paldi A, Dezsö L, Varga L, Magyar A. Prenatal fate of parthenogenetic cells in mouse aggregation chimeras. Development 1987 ; 101: 67-71.

13. Fundele R, Norris ML, Barton SC, Reik W, Surani MAH. Systematic elimination of parthenogenetic cells in mouse chimeras. Development 1989; 106 : 29-35.

14. Nagy A, Sass M, Markkula M. Systematic non uniform distribution of parthenogenetic cells in adult mouse chimeras. Development $1989 ; 106: 321-4$.

15. Paldi A, Nagy A, Markkula $M$, Barna I, Dezsö L. Postnatal development of parthenogenetic $\leftrightarrow$ fertilized mouse aggregation chimeras. Development $1989 ; 105$ : 115-8.

16. Fundele R, Norris ML, Barton SC, Fehlau M, Howlett SK, Mills WE, Surani MAH. Temporal and spatial selection against parthenogenetic cells during development of fetal chimeras. Development 1990 ; 108: 203-11.

17. Mann JR, Gadi I, Harbison ML, Abbondanzo SJ, Stewart CL. Androgenetic mouse embryonic stem cells are pluripotent and cause skeletal defects in chimeras : implications for genetic imprinting. Cell $1990 ; 62: 251-60$.

18. Robertson EJ. Pluripotential stem cell lines as a route into mouse germ line. Trends Genet $1986 ; 2$ : 9-13.

19. Searl AG, Beechey CV. Complementation studies with mouse translocations. Cytogenet Cell Genet 1978; 20: 222-303.

20. Cattanach BM. Parental origin effects in mice. J Embryol Exp Morphol 1986 ; 97 (suppl) : 137-50.

21. Johnson DR. Hairpin-tail : a case of post-reductional gene action in the mouse egg ? Genetics $1974 ; 76$ : 795-805.

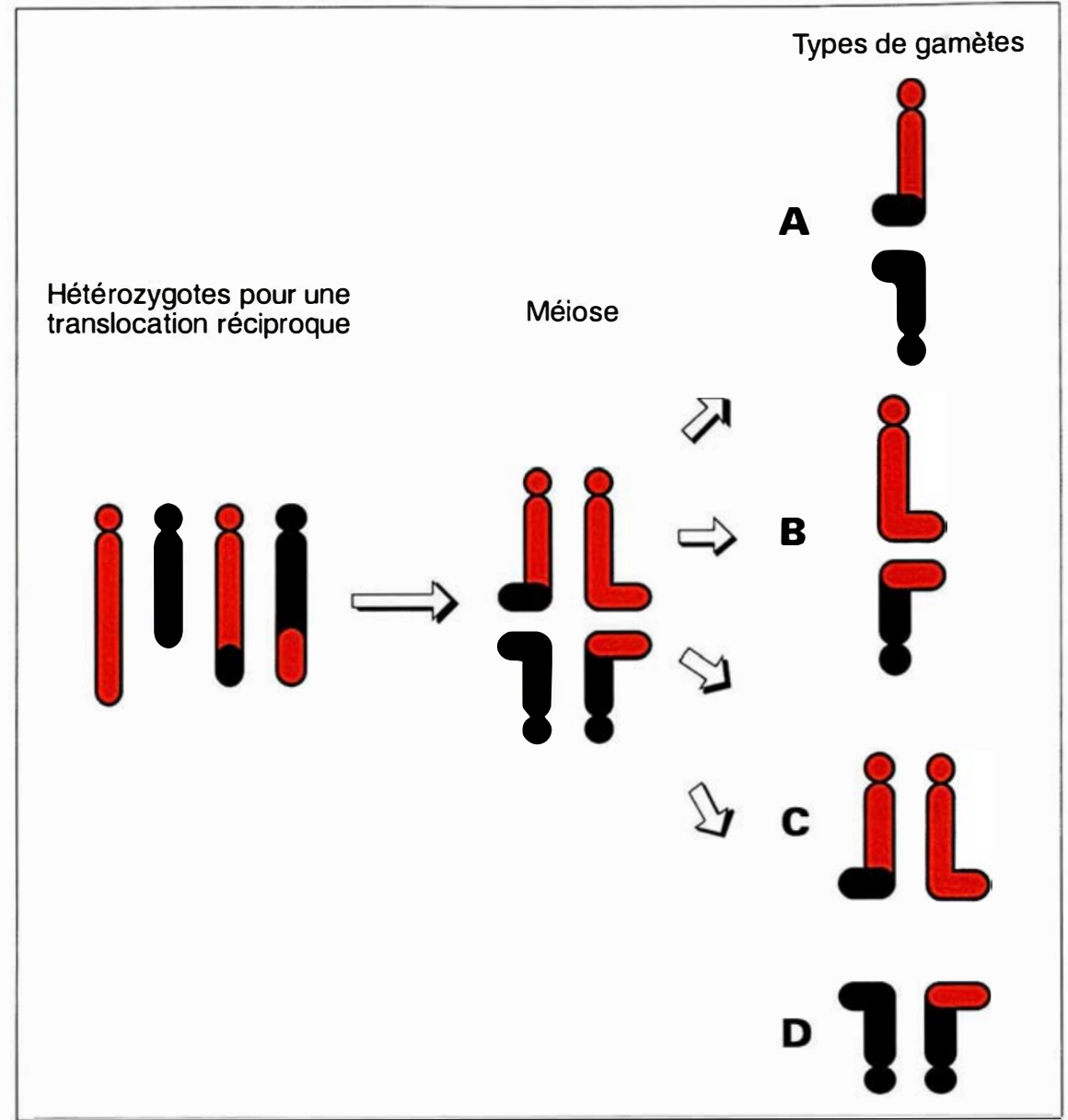

Figure 5. Mécanisme de la constitution d'animaux diploïdes porteurs de régions chromosomiques dont les deux copies proviennent du même parent. Un animal hétérozygote pour une translocation réciproque impliquant deux chromosomes (rouge et noir) a un complément chromosomique équilibré. La répartition des chromosomes lors de la méiose aboutit à la constitution de gamètes pouvant contenir la copie d'un seul des deux chromosomes portant une translocation, $c^{\prime} e s t$-à-dire portant une duplication d'une région et dépourvus d'une autre région chromosomique. La combinaison de deux gamètes complémentaires lors du croisement de deux hétérozygotes rétablit la disomie, mais les deux copies des fragments chromosomiques impliqués dans la translocation réciproque proviennent soit du père soit de la mère. Les gamètes $A$ sont nullisomiques pour la partie télomérique du chromosome rouge et disomiques pour celle du chromosome noir. Les gamètes $B$ sont nullisomiques pour la partie télomérique du chromosome noir et disomiques pour celle du chromosome rouge. Les mêmes remarques s'appliquent pour la partie centromérique des chromosomes des gamètes $C$ et $D$. Les diploïdes issus des croisements $A \times B$ seront disomiques uniparentaux pour les régions télomériques noires et rouges, alors que ceux issus des croisements $C \times D$ le seront pour les régions centromériques des deux mêmes chromosomes. 


\begin{tabular}{|c|c|c|}
\hline D'UNE & $\begin{array}{l}\text { Tableau I } \\
\text { FET DE L'ORIGINE UNIP } \\
\text { JION CHROMOSOMIQUE }\end{array}$ & $\begin{array}{l}\text { NTALE } \\
\text { EZ LA SOURIS }\end{array}$ \\
\hline Chromosome $n^{\circ}$ & $\begin{array}{l}\text { Duplication maternelle/ } \\
\text { absence paternelle }\end{array}$ & $\begin{array}{l}\text { Duplication paternelle/ } \\
\text { absence maternelle }\end{array}$ \\
\hline $\begin{array}{l}2 \text { distal } \\
2 \text { proximal } \\
6 \\
7 \text { proximal } \\
7 \\
8 \\
8 \text { distal } \\
11 \\
12\end{array}$ & $\begin{array}{c}\text { hypokinésie } \\
\text { létal } \\
\text { létal } \\
\text { létal } \\
? \\
\text { létal } \\
\text { petite taille } \\
\text { viable }\end{array}$ & $\begin{array}{c}\text { hyperkinésie } \\
\text { viable } \\
\text { viable } \\
? \\
\text { létal } \\
? \\
\text { grande taille } \\
\text { létal }\end{array}$ \\
\hline
\end{tabular}

tion paternelle/déficience maternelle de la même région est parfaitement viable. D'autres exemples sont présentés dans le Tableau I. Dans d'autres cas, la " non-complémentation " n'est pas totale et on observe les phénotypes complémentaires qui sont tous deux viables. Par exemple, quel que soit leur sexe, les souris porteuses d'une duplication maternelle/déficience paternelle du chromosome 11 sont de très petite taille, tandis qu'au contraire les souris avec la duplication paternelle/déficience maternelle sont deux fois plus grandes que les souris normales. Dans les deux cas, tous les descendants de ces souris issus d'un croisement entre un frère et une sœur sont de taille normale [2]. La réciprocité des phénotypes et leur disparition à la génération suivante souligne une fois de plus la complémentarité fonctionnelle des deux génomes parentaux et la réversibilité de l'empreinte parentale d'une génération à l'autre.

\section{Approches visant à identifier les gènes soumis à l'empreinte parentale}

Les études embryologiques et génétiques donnent peu de points de repère pour identifier des gènes porteurs d'une empreinte parentale. Une approche génétique classique serait l'étude de mutants. Mais les mutations chez la souris dont l'expression phénotypique dépend de la transmission paternelle ou maternelle sont extrêmement rares. Deux mutations seulement sont connues pour obéir de l'ADN localisées autour du site d'insertion et qui sont l'objet d'une empreinte parentale révélée par celle subie par le transgène.

\section{Mécanisme moléculaire de l'empreinte parentale}

Malgré notre ignorance de la nature des gènes soumis à l'empreinte parentale, les observations faites sur des lignées de souris transgéniques nous donnent une idée de la complexité du mécanisme génétique. Puisque certains transgènes obéissent aux règles de l'empreinte parentale, on peut supposer qu'ils sont modifiés par le même mécanisme moléculaire que les gènes endogènes soumis à ce phénomène [26-29]. Un tel mécanisme doit répondre à certains critères. Il doit affecter l'expression des gènes, être physiquement lié à l'ADN, être transmis par une cellule à ses descendantes et être réversible, cette réversion se faisant lors de son passage dans la lignée germinale. La méthylation de l'ADN peut satisfaire ces conditions. En effet, l'examen des lignées décrites précédemment suggère qu'il existe une corrélation entre le degré de méthylation, l'expression et l'origine parentale du transgène. L'expression est toujours accompagnée d'une hypométhylation. Le niveau d'expression comme la méthylation ne dépendent pas du sexe de l'animal mais uniquement du sexe du parent transmetteur. Des degrés différents de méthylation peuvent donc être corrélés à des niveaux d'expression différents des transgènes, mais nous ne savons pas encore si la méthylation est la cause ou seulement la conséquence de l'inactivation du gène. L'exemple du chromosome $\mathrm{X}$ suggère que c'est la deuxième hypothèse qui est correcte : tous les gènes situés sur le chromosome $\mathrm{X}$ qui ont été jusqu'ici analysés sont méthylés seulement après l'inactivation de ce chromosome [5]. Il est donc probable que la méthylation stabilise plutôt qu'clle n'induit l'inactivation du gène.

Il faut ajouter que l'origine parentale n'est pas le scul facteur influençant le niveau d'expression et le degré de méthylation des transgènes dans les lignées décrites. Le mécanisme de l'empreinte parentale elle-même est 


\section{RÉFÉRENCES}

22. Johnson DR. Further observations on the hairpin tail ( $T^{\mathrm{h} p}$ ) mutation in the mouse. Genet Res 1975; 24 : 207-13.

23. Winking $\mathrm{H}$, Silver LM. Characterization of a recombinant mouse $t$ haplotype that expresses a dominant lethal maternal effect. Genetics 1984; 108 : 1013-20.

24. Paldi A, Jami J, Tcp-1 is not responsible for the maternal lethality cffect in $T^{\text {hp }}$ p mutant mice. Dev Biol 1991 (sous presse).

25. DeLoia J, Solter D. Effect of imprinting on analysis of genetic mutations. In : Banbury Report 34. Biology of Mammalian Germ Cell Mutagenesis. Cold Spring Harbor Lab Press, 1990 : 393-8.

26. Swain JL, Stewart TA, Leder P. Parental legacy determines methylation and expression of an autosomal transgene : a molecular mechanism for parental imprinting. Cell $1987 ; 50: 719-27$.

27. Reik W, Collick A, Norris ML, Barton SC, Surani MAH. Genomic imprinting determines methylation of parental alleles in transgenic mice. Nature 1987 ; 328 : 248-51.

28. Sapienza C, Peterson AC, Rossant J, Balling $R$. Degree of methylation of transgenes is dependent on gamete origin. Nalure 1987 ; 328 : 251-4.

29. Hadchouel M, Farza H, Simon D, Tiollais P, Pourcel C. Maternal inhibition of hepatitis B surface antigen gene expression in transgenic mice correlates with de novo methylation. Nature 1987 ; 329 : 454-6.

30. Allen ND, Norris ML, Surani MAH. Epigenetic control of transgene expression and imprinting by genotype specific modifiers. Cell $1990 ; 61$ : 853-61.

31. Junien C, Henry I. Bras court du chromosome 11 : empreinte parentale différentielle, tumorigenèse et pertes d'allèles. médecine/sciences $1989 ; 5$ : 480-8.

32. Reik W. Genomic iniprinting and genetic disorders in man. Trends Genet 1989; 5 : $331-6$. probablement sous contrôle génétique, l'environnement génétique interférant avec l'existence ou la manifestation de l'empreinte parentale. Par exemple, dans une lignée de souris transgéniques, le niveau de l'expression du transgène devient plus élevé et son degré de méthylation plus bas quand les souris transgéniques sont croisées avec des souris de souche DBA/2 ou 129 [30]. C'est exactement le contraire qui se produit quand les mâles transgéniques sont croisés avec des femelles BALB/c : l'expression diminue et le degré de méthylation augmente. Le croisement réciproque femelle transgénique $x$ mâle BALB/c n'a pas cet effet. Ces observations ont amené les chercheurs à supposer l'existence de gènes modificateurs qui influenceraient l'activité et la méthylation d'autres gènes, et qui eux-mêmes seraient soumis à une empreinte parentale. Ces gènes régulateurs, dont nous ignorons le nombre et la nature, peuvent avoir plusieurs allèles. La composition allélique (haplotype) de chaque lignée consanguine, celle de chaque individu d'une population sauvage, peut être différente. Cela signifierait que chaque individu porterait une empreinte génétique particulière, déterminée par les allèles régulateurs de ses parents. Les données présentées dans cet article proviennent des études effectuées sur la souris de laboratoire. Mais il semble bien que l'empreinte parentale existe aussi chez les autres mammifères, y compris dans l'espèce humaine. Comme nous le voyons, c'est un phénomène très complexe et nous sommes encore loin de le comprendre. Il s'agit probablement d'un nouveau mode de régulation et de fonctionnement du génome des mammifères, qui pourrait permettre d'expliquer certaines maladies héréditaires ou acquises [31, 32 et $\mathrm{m} / \mathrm{s}$ $n^{\circ} 1$, vol. 6, p. 57]

\section{Remerciements}

Les auteurs expriment leur gratitude à B. Maro, L. Deltour et M.-A. Ripoche pour les discussions.

\section{Summary}

Genomic imprinting : functional complementarity of the two parental genomes

The parental and maternal genomes are not equivalent and have a complementary role during development in mammals. The differences in activity of each parental genome result from an epigenetic modification - the parental imprinting - of the genome during gametogenesis. The yield of gene imprinting remains so far undetermined, and only a single gene submitted to this process has recently been identified. Parental imprinting was mainly deduced from the limited and complementary developmental capacities of uniparental mouse embryos. The maternal genome is necessary to embryo development. The paternal genome is required for the full growth and differentiation of extraembryonic tissues. The biological results of parental imprinting can be observed in mice carrying chromosomal translocations with the two copies of a chromosomal fragment originating from a single parent. The observed phenotypes indicate that several chromosomal regions are not functionally equivalent depending on the maternal or paternal origin of the chromosomal fragments. The random integration of transgenes into the chromosomes of the mouse has revealed other chromosomal regions submitted to parental imprinting, and will allow the cloning of the genes involved in the process. High methylation of the transgene is correlated with its non-expression. Genomic imprinting is a novel regulatory mechanism of gene expression that might account for some hereditary or acquired diseases.

\section{TIRÉS A PART}

J. Jami.

$\mathrm{m} / \mathrm{s} n^{\circ} 3$, vol. 7, mars 91 